\title{
Strong Scalar QED in Inhomogeneous Electromagnetic Fields
}

\author{
Sang Pyo Kin* \\ Department of Physics, Kunsan National University, Kunsan 573-701, Korea and \\ Asia Pacific Center for Theoretical Physics, Pohang 790-784, Korea
}

(Dated:)

\begin{abstract}
Strong QED has attracted attention recently partly because many astrophysical phenomena have been observed to involve electromagnetic fields beyond the critical strength for electron-positron pair production and partly because terrestrial experiments will generate electromagnetic fields above or near the critical strength in the near future. In this talk we critically review QED phenomena involving strong external electromagnetic fields. Strong QED is characterized by vacuum polarization due to quantum fluctuations and pair production due to the vacuum instability. A canonical method is elaborated for pair production at zero or finite temperature by inhomogeneous electric fields. An algorithm is advanced to calculate pair production rate for electric fields acting for finite periods of time or localized in space or oscillating electric fields. Finally, strong QED is discussed in astrophysics, in particular, strange stars.

Keywords: Strong QED, Inhomogeneous Electromagnetic field, Schwinger pair production, Strange star

PACS numbers: 12.20.-m, 13.40.-f, 11.10.Wx
\end{abstract}

\section{INTRODUCTION}

Recently strong QED (quantum electrodynamics) has attracted much attention not only from theoretical interest but also from astrophysical observations and terrestrial experimental tests in the near future. From a theoretical view point, calculating the full nonperturbative effective action under the influence of strong external electromagnetic fields, in particular, inhomogeneous fields, is still a challenging task (for a recent review and references, see Ref. 11] and also Ref. 2]). From an experimental view point, in the near future electromagnetic fields from X-ray free electron lasers from LCLS (Linac Coherent Light Source) at SLAC [3] and TESLA (TeV Energy Superconducting Linear Accelerator) at DESY [4] may attain a strength almost comparable to the critical value for electron-positron pair production, which will directly test strong QED [5]. Interestingly, astrophysical sources have been predicted and observed that can have electromagnetic fields greater than the critical strength. Neutron stars have magnetic fields ranging from $10^{8} \mathrm{G}$ to $10^{15} \mathrm{G}$ and more than one-tenth of them have magnetic fields stronger than $10^{14} \mathrm{G}$, the so-called magnetars (for a review and references, see Ref. [6]), at least one order greater than the critical strength. Another interesting astrophysical objects with a ultra-strong electromagnetic field are strange quark stars, hypothetical objects, which may have electric fields with one or two order higher than the critical strength [7, 8] (see also Ref. [9] for review and references).

Vacuum fluctuations due to a strong external electromagnetic field contribute nonlinear terms to the classical Maxwell theory and the electromagnetic theory thus

*Electronic address: sangkim@kunsan.ac.kr becomes highly nonlinear. Physics in strong electromagnetic fields drastically differs from the Maxwell theory [10, 11]. The cyclotron energy of an electron in a strong magnetic field can be greater than the rest mass energy of electron, the equivalent value leading to the critical strength of magnetic field $B_{c}=m^{2} c^{3} / e \hbar\left(4.4 \times 10^{13} \mathrm{G}\right)$. Similarly, in a strong electric field, virtual pairs of electrons and positrons can gain energy comparable to or greater than the rest mass energy of electron or positron. The electric field whose potential energy across the Compton wavelength is the rest mass energy of electron is the critical value $E_{c}=m^{2} c^{3} / e \hbar\left(2.2 \times 10^{15} \mathrm{~V} / \mathrm{cm}\right)$. For magnetic fields greater than the critical value, nonlinear contributions to the Maxwell term make the vacuum polarized by quantum fluctuations and the vacuum polarization causes nonlinear effects such as birefringence (propagation of photons in the magnetic vacuum), which plays an important role in the physics of magnetars [10]. For strong electric fields the vacuum decays due to an imaginary part of the effective action and thus leads to Schwinger pair production [12]. Strange stars can emit electron-positron pairs more efficiently than photons [13, 14, 15]. On the other hand, in the standard QED with the minimal interaction magnetic fields are stable up to $B=10^{32} \mathrm{G}$ due to the instability from the self-interaction of an electron and up to the range $B=10^{51}-10^{55} \mathrm{G}$ due to the instability from magnetic monopole production at the string or Planck scale [16]. However, the Pauli interaction may open a window for pair production by a far weaker inhomogeneous magnetic field and would have astrophysical applications [17].

QED describes the interaction between charged particles and photons. The success of QED is based on the perturbation theory in the weak-field limit. However, QED has not been completely understood yet in the opposite case of strong electromagnetic fields partly because the full nonperturbative QED action is not known except for some exactly solved cases [1, 2]. Historically, the 
effective action of an electron in a constant electromagnetic field was obtained by Heisenberg and Euler [18], and also by Weisskopf [19]. Using the proper time method, Schwinger found the one-loop effective action for a spin$1 / 2$ fermion with charge $q$ and mass $m$ in a constant electromagnetic field [12]

$$
\begin{aligned}
\mathcal{L}_{\text {eff }}= & -\mathcal{F}-\frac{1}{8 \pi^{2}} \int_{0}^{\infty} d s \frac{e^{-m^{2} s}}{s^{3}} \\
& \times\left[(q s)^{2} \mathcal{G} \frac{\operatorname{Re} \cosh (q s X)}{\operatorname{Im} \cosh (q s X)}-1-\frac{2}{3}(q s)^{2} \mathcal{F}\right] .
\end{aligned}
$$

where

$$
X=[2(\mathcal{F}+i \mathcal{G})]^{1 / 2}=X_{r}+i X_{i} .
$$

Here, $\mathcal{F}$ is the negative of the Maxwell term, $-\mathcal{L}_{\text {Maxwell }}$,

$$
\mathcal{F}=\frac{1}{4} F_{\mu \nu} F^{\mu \nu}=\frac{1}{2}\left(\mathbf{B}^{2}-\mathbf{E}^{2}\right),
$$

and $\mathcal{G}$ is another Lorentz invariant tensor

$$
\mathcal{G}=\frac{1}{4} F_{\mu \nu} \tilde{F}^{\mu \nu}=\mathbf{E} \cdot \mathbf{B}
$$

where $\tilde{F}^{\mu \nu}=\epsilon^{\mu \nu \alpha \beta} F_{\alpha \beta}$ is the dual field tensor. The oneloop effective action was also obtained in Ref. [20].

The one-loop effective action has two important aspects. First, in the weak-field limit the nonlinear contribution to the real part

$$
\operatorname{Re} \mathcal{L}^{(1)}=\frac{2}{45 m c^{2}}\left(\frac{q^{2}}{4 \pi \hbar}\right)^{2}\left(\frac{\hbar}{m c}\right)^{3}\left(4 \mathcal{F}^{2}+7 \mathcal{G}^{2}\right),
$$

makes the vacuum polarized by quantum fluctuations. In a pure strong magnetic field the leading term becomes

$$
\mathcal{L}^{(1)}=\frac{(q B)^{2}}{24 \pi^{2}} \ln \left(\frac{2 q B}{m^{2}}\right) .
$$

The ratio $\mathcal{L}^{(1)} / \mathcal{L}_{\text {Maxwell }}=-\left(q^{2} / 12 \pi^{2}\right) \ln \left(q B / m^{2}\right)$ is the one-loop QED $\beta$-function related with the renormalization group [1]. Second, in spinor QED in a pure strong electric field pairs are produced at the rate per unit time and unit volume

$$
w^{\text {fermion }}=2 \operatorname{Im} \mathcal{L}_{\text {eff }}^{\text {fermion }}=\frac{2}{(2 \pi)^{3}} \sum_{n=1}^{\infty}\left(\frac{q E}{n}\right)^{2} e^{-\frac{n \pi m^{2}}{q E}},
$$

and in scalar QED pairs at the rate

$w^{\text {boson }}=2 \operatorname{Im} \mathcal{L}_{\text {eff }}^{\text {boson }}=\frac{2}{(2 \pi)^{3}} \sum_{n=1}^{\infty}(-1)^{n+1}\left(\frac{q E}{n}\right)^{2} e^{-\frac{n \pi m^{2}}{q E}}$.

Here, the factor of 2 is the spin multiplicity. The scattering amplitude of the ingoing vacuum to the outgoing vacuum decays according to Eqs. (7) or (8), leading to Schwinger pair production. One way to understand Schwinger pair production is to compute the imaginary part of the effective action. Another way is to find the vacuum solution of the field equation and then calculate the number of pairs produced by the field. The tunneling interpretation is that charged pairs in the Dirac sea can tunnel quantum mechanically through the potential barrier lowered by a uniform electric field. Pair production is an efficient mechanism for energy extraction from objects with strong electromagnetic fields.

In this talk, within the framework of canonical quantum field theory we critically review the Schwinger mechanism at zero or finite temperature in inhomogeneous electric fields motivated by terrestrial experiments or astrophysics. Exact solutions of the Klein-Gordon or Dirac equation minimally coupled to inhomogeneous electromagnetic fields are known only for a few models. The Sauter-type electric field that extends for a finite region or lasts for a finite period is the most well-known model 21]. However, one cannot find, in general, solutions for arbitrary electromagnetic fields, so he or she has to employ some approximation schemes. It is known that pair production by a constant electric field that extend over a finite region has a finite size effect and differs from that by a constant field [22]. Also the pair production rate by a Sauter-type electric field obtained by the worldline instanton method depends on the characteristic scale in a nontrivial way [23, 24]. Applying the phase-integral method [25] to find the WKB instanton action for the field equation with an electric and/or magnetic field in a fixed direction, the pair production rate is obtained for the Sauter-type electric field either in space or time with or without a constant magnetic field [26, 27, 28]. Further, a perturbative method is advanced to calculate the WKB instanton action for pair production by any analytical electric field and is then applied to strange stars to calculate the production rate of electron-positron pairs. The thermal effect on the pair production is also studied [29].

The organization of this talk is as follows. In Sec. II, we critically review the Schwinger mechanism and then thermal effects on pair production. In Secs. III and IV, we apply the WKB instanton action method to inhomogeneous electric fields that act on for a finite period or extend for a finite region or oscillate. In Sec. IV, we apply strong QED to calculate the pair production rate from strange stars.

\section{CANONICAL METHOD FOR PAIR PRODUCTION}

In real physical systems electric fields are either confined to finite regions or turned on for finite periods of time. For such electric fields it is a nontrivial task to calculate the pair production rate. Instead of applying the proper time method or path integral method, we employ an approximation scheme such as the WKB approximation and phase integral in canonical quantum field theory. For the sake of convenience we consider only scalar QED, but the formalism here can be directly applied to spinor 
QED [27, 28].

\section{A. Schwinger Pair Production}

The Klein-Gordon equation for a charged boson with $q(q>0)$ and $m$ takes the form (in units with $\hbar=c=1$ and with metric signature $(+,-,-,-))$

$$
\left[\eta^{\mu \nu}\left(\partial_{\mu}+i q A_{\mu}\right)\left(\partial_{\nu}+i q A_{\nu}\right)+m^{2}\right] \Phi(\mathbf{x}, t)=0 .
$$

Hereafter we further restrict our study to time-dependent electric fields along the $z$ direction with gauge potentials of the form $A_{z}(t)=-E_{0} g(t)$ for any analytic function $g(t)$. Then the Fourier mode, $\Phi(\mathbf{x}, t)=e^{i \mathbf{k} \cdot \mathbf{x}} \phi_{\mathbf{k}}$, satisfies

$$
\left[\frac{\partial^{2}}{\partial t^{2}}+m^{2}+\mathbf{k}_{\perp}^{2}+\left(k_{z}+q E_{0} g(t)\right)^{2}\right] \varphi_{\mathbf{k}}(t)=0 .
$$

The solution can be used to quantize the position operators as

$$
\begin{aligned}
& \hat{\phi}_{\mathbf{k}}(t)=\varphi_{\mathbf{k}}(t) \hat{a}_{\mathbf{k}}(t)+\varphi_{\mathbf{k}}^{*}(t) \hat{b}_{\mathbf{k}}^{\dagger}(t), \\
& \hat{\phi}_{\mathbf{k}}^{*}(t)=\varphi_{\mathbf{k}}(t) \hat{b}_{\mathbf{k}}(t)+\varphi_{\mathbf{k}}^{*}(t) \hat{a}_{\mathbf{k}}^{\dagger}(t),
\end{aligned}
$$

and the momentum operators as

$$
\begin{aligned}
& \pi_{\mathbf{k}}(t)=\dot{\varphi}_{\mathbf{k}}^{*}(t) a_{\mathbf{k}}^{\dagger}(t)+\dot{\varphi}_{\mathbf{k}}(t) b_{\mathbf{k}}(t), \\
& \pi_{\mathbf{k}}^{*}(t)=\dot{\varphi}_{\mathbf{k}}^{*}(t) b_{\mathbf{k}}^{\dagger}(t)+\dot{\varphi}_{\mathbf{k}}(t) a_{\mathbf{k}}(t) .
\end{aligned}
$$

On the other hand, in quantum mechanics, Eq. (10) is a one-dimensional scattering problem with inverted potential. The positive (asymptotic) solution $\varphi_{\mathbf{k} \text {,in }}$ at one asymptotic region $t=-\infty$ defines the (asymptotic) ingoing vacuum and another positive (asymptotic) solution $\varphi_{\mathbf{k} \text {,out }}$ at the other region $t=\infty$ defines the (asymptotic) outgoing vacuum. As an incident solution from $t=\infty$ is partially transmitted over the barrier to $t=-\infty$ and partially reflected by the barrier back to $t=\infty$, the ingoing solution is related with the outgoing solution as

$$
\varphi_{\mathbf{k}, \text { in }}=\mu_{\mathbf{k}} \varphi_{\mathbf{k}, \text { out }}+\nu_{\mathbf{k}} \varphi_{\mathbf{k}, \text { out }}^{*} .
$$

That is, the ingoing positive frequency solution is mixed both with the outgoing positive solution and with the outgoing negative solution, which is the origin of particle production by an external field [30, 31]. As the Wronskian

$$
\dot{\varphi}_{\mathbf{k}}^{*}(t) \varphi_{\mathbf{k}}(t)-\dot{\varphi}_{\mathbf{k}}(t) \varphi_{\mathbf{k}}^{*}(t)=i,
$$

is constant, the coefficients satisfy the relation

$$
\left|\mu_{\mathbf{k}}\right|^{2}-\left|\nu_{\mathbf{k}}\right|^{2}=1 .
$$

In fact, the annihilation and creation operators at two asymptotic regions are related through Bogoliubov transformations

$$
\begin{aligned}
& \hat{a}_{\mathbf{k}, \text { in }}=\mu_{\mathbf{k}}^{*} \hat{a}_{\mathbf{k}, \text { out }}-\nu_{\mathbf{k}}^{*} \hat{b}_{\mathbf{k}, \text { out }}^{\dagger}, \\
& \hat{b}_{\mathbf{k}, \text { in }}=\mu_{\mathbf{k}}^{*} \hat{b}_{\mathbf{k}, \text { out }}-\nu_{\mathbf{k}}^{*} \hat{a}_{\mathbf{k}, \text { out }}^{\dagger} .
\end{aligned}
$$

The inverse Bogoliubov transformations are

$$
\begin{aligned}
& \hat{a}_{\mathbf{k}, \text { out }}=\mu_{\mathbf{k}} \hat{a}_{\mathbf{k}, \text { in }}+\nu_{\mathbf{k}}^{*} \hat{b}_{\mathbf{k}, \text { in }}^{\dagger}, \\
& \hat{b}_{\mathbf{k}, \text { out }}=\mu_{\mathbf{k}} \hat{b}_{\mathbf{k}, \text { in }}+\nu_{\mathbf{k}}^{*} \hat{a}_{\mathbf{k}, \text { in }}^{\dagger} .
\end{aligned}
$$

Therefore, the outgoing vacuum contains the ingoing particles/antiparticles as 30, 31

$$
\begin{aligned}
\left\langle 0, \text { out }\left|\sum_{\mathbf{k}} \hat{a}_{\mathbf{k}, \text { in }}^{\dagger} \hat{a}_{\mathbf{k}, \text { in }}\right| 0, \text { out }\right\rangle & =\sum_{\mathbf{k}}\left|\nu_{\mathbf{k}}\right|^{2}, \\
\left\langle 0, \text { out }\left|\sum_{\mathbf{k}} \hat{b}_{\mathbf{k}, \text { in }}^{\dagger} \hat{b}_{\mathbf{k}, \text { in }}\right| 0, \text { out }\right\rangle & =\sum_{\mathbf{k}}\left|\nu_{\mathbf{k}}\right|^{2},
\end{aligned}
$$

and, conversely, the ingoing vacuum evolves into outgoing particle/antiparticle states as

$$
\begin{aligned}
\left\langle 0, \text { in }\left|\sum_{\mathbf{k}} \hat{a}_{\mathbf{k}, \text { out }}^{\dagger} \hat{a}_{\mathbf{k}, \text { out }}\right| 0, \text { in }\right\rangle & =\sum_{\mathbf{k}}\left|\nu_{\mathbf{k}}\right|^{2}, \\
\left\langle 0, \text { in }\left|\sum_{\mathbf{k}} \hat{b}_{\mathbf{k}, \text { out }}^{\dagger} \hat{b}_{\mathbf{k}, \text { out }}\right| 0, \text { in }\right\rangle & =\sum_{\mathbf{k}}\left|\nu_{\mathbf{k}}\right|^{2} .
\end{aligned}
$$

\section{B. Hamiltonian Approach}

Pair production by time-dependent electric fields can also be described by the Hamiltonian formalism. The Hamiltonian formalism is particulary appropriate for studying thermal effects because the density operator should satisfy the Liouville-von Neumann equation with respect to the Hamiltonian itself. The complex scalar field has the Hamiltonian

$$
H=\int[d \mathbf{k}]\left[\pi_{\mathbf{k}}^{*} \pi_{\mathbf{k}}+\omega_{\mathbf{k}}^{2}(t) \phi_{\mathbf{k}}^{*} \phi_{\mathbf{k}}\right],
$$

where $[d \mathbf{k}]=d^{3} \mathbf{k} /(2 \pi)^{3}$ and

$$
\omega_{\mathbf{k}}^{2}(t)=m^{2}+\mathbf{k}_{\perp}^{2}+\left(k_{z}+q E_{0} g(t)\right)^{2} .
$$

The field operators are quantized as

$$
\begin{aligned}
\hat{\phi}(t, \mathbf{x}) & =\int[d \mathbf{k}]\left[\varphi_{\mathbf{k}}(t) \hat{a}_{\mathbf{k}}(t)+\varphi_{\mathbf{k}}^{*}(t) \hat{b}_{\mathbf{k}}^{\dagger}(t)\right] e^{i \mathbf{k} \cdot \mathbf{x}}, \\
\hat{\phi}^{*}(t, \mathbf{x}) & =\int[d \mathbf{k}]\left[\varphi_{\mathbf{k}}(t) \hat{b}_{\mathbf{k}}(t)+\varphi_{\mathbf{k}}^{*}(t) \hat{a}_{\mathbf{k}}^{\dagger}(t)\right] e^{-i \mathbf{k} \cdot \cdot x}
\end{aligned}
$$

and the momentum operators as

$$
\begin{aligned}
\hat{\pi}(t, \mathbf{x}) & =\int[d \mathbf{k}]\left[\dot{\varphi}_{\mathbf{k}}^{*}(t) \hat{a}_{\mathbf{k}}^{\dagger}(t)+\dot{\varphi}_{\mathbf{k}}(t) \hat{b}_{\mathbf{k}}(t)\right] e^{-i \mathbf{k} \cdot \mathbf{x}}, \\
\hat{\pi}^{*}(t, \mathbf{x}) & =\int[d \mathbf{k}]\left[\dot{\varphi}_{\mathbf{k}}^{*}(t) \hat{b}_{\mathbf{k}}^{\dagger}(t)+\dot{\varphi}_{\mathbf{k}}(t) \hat{a}_{\mathbf{k}}(t)\right] e^{i \mathbf{k} \cdot \mathbf{x}} \cdot(2)
\end{aligned}
$$

In the Hamiltonian approach both quantum states and the density operator can be found simultaneously using the operators that satisfy the Liouville-von Neumann equation [32, 33]

$$
i \frac{\partial \hat{\rho}_{\mathbf{k}}(t)}{\partial t}+\left[\hat{\rho}_{\mathbf{k}}(t), \hat{H}(t)\right]=0 .
$$


In fact, there are the time-dependent annihilation and creation operators satisfying Eq. (24) for particles

$$
\begin{aligned}
& \hat{a}_{\mathbf{k}}(t)=i\left[\varphi_{\mathbf{k}}^{*}(t) \hat{\pi}_{\mathbf{k}}^{*}-\dot{\varphi}_{\mathbf{k}}^{*}(t) \hat{\phi}_{\mathbf{k}}\right], \\
& \hat{a}_{\mathbf{k}}^{\dagger}(t)=-i\left[\varphi_{\mathbf{k}}(t) \hat{\pi}_{\mathbf{k}}-\dot{\varphi}_{\mathbf{k}}(t) \hat{\phi}_{\mathbf{k}}^{*}\right],
\end{aligned}
$$

and for antiparticles

$$
\begin{aligned}
& \hat{b}_{\mathbf{k}}(t)=i\left[\varphi_{\mathbf{k}}^{*}(t) \hat{\pi}_{\mathbf{k}}-\dot{\varphi}_{\mathbf{k}}^{*}(t) \hat{\phi}_{\mathbf{k}}^{*}\right] \\
& \hat{b}_{\mathbf{k}}^{\dagger}(t)=-i\left[\varphi_{\mathbf{k}}(t) \hat{\pi}_{\mathbf{k}}^{*}-\dot{\varphi}_{\mathbf{k}}(t) \hat{\phi}_{\mathbf{k}}\right] .
\end{aligned}
$$

Then the time-dependent vacuum, an exact state of the time-dependent Schrödinger equation, is given by

$$
\left.\hat{a}_{\mathbf{k}}(t)|0 ; t\rangle=\hat{b}_{\mathbf{k}}(t)|0 ; t\rangle=0 \quad \text { (for any } \mathbf{k}\right),
$$

and multi-particle and antiparticle states by

$$
\left|n_{\mathbf{k}_{1}} \cdots ; n_{\mathbf{k}_{2}} \cdots ; t\right\rangle=\frac{\hat{a}_{\mathbf{k}_{1}}^{\dagger n_{1}}(t)}{\sqrt{n_{1} !}} \cdots \frac{\hat{b}_{\mathbf{k}_{2}}^{\dagger n_{2}}(t)}{\sqrt{n_{2} !}} \cdots|0 ; t\rangle .
$$

\section{Pair Production at Finite Temperature}

The finite temperature QED effective action was calculated in a constant magnetic field [34], a constant electromagnetic field [35], and at finite density [36]. The Schwinger proper-time method was used to derive the effective action in a constant electromagnetic field, which exhibits the Schwinger mechanism at high temperature [37]. However, depending on the formalism employed to calculate the effective action, pairs are either produced [38, 39] or not produced [40]. The QED effective action from the imaginary-time formalism has nonzero imaginary part at two-loop [41]. In this paper we follow the real-time formalism in Ref. 29] to obtain pair production at finite temperature at one-loop.

As $\hat{a}_{\mathbf{k}}(t), \hat{a}_{\mathbf{k}}^{\dagger}$ and $\hat{b}_{\mathbf{k}}(t), \hat{b}_{\mathbf{k}}^{\dagger}$ satisfy Eq. (24), the density operator for particles can be found as 33 ]

$$
\hat{\rho}_{a_{\mathbf{k}}}(t)=\frac{1}{Z_{\mathbf{k}}} \exp \left[-\beta \omega_{\mathbf{k}}^{\mathrm{in}}\left(\hat{a}_{\mathbf{k}}^{\dagger}(t) \hat{a}_{\mathbf{k}}(t)+\frac{1}{2}\right)\right],
$$

with $\beta=1 /(k T)$ is the inverse temperature, and for antiparticles as

$$
\hat{\rho}_{b_{\mathbf{k}}}(t)=\frac{1}{Z_{\mathbf{k}}} \exp \left[-\beta \omega_{\mathbf{k}}^{\text {in }}\left(\hat{b}_{\mathbf{k}}^{\dagger}(t) \hat{b}_{\mathbf{k}}(t)+\frac{1}{2}\right)\right] .
$$

Then the pair production rate for each spin component from an initial ensemble by an electric field acting for a finite period of time is given by [29]

$$
\begin{aligned}
n_{\mathbf{k}}(E, T) & =\operatorname{Tr}\left(\hat{\rho}_{\mathbf{k}}^{\text {in }} \hat{N}_{a_{\mathbf{k}}}^{\text {out }}\right)-f_{\mathbf{k}}^{\text {in }} \\
& =\left|\nu_{\mathbf{k}}(E)\right|^{2}\left(2 f_{\mathbf{k}}^{\text {in }}(T)+1\right),
\end{aligned}
$$

where $f_{\mathbf{k}}^{\text {in }}$ is the Bose-Einstein distribution

$$
f_{\mathbf{k}}^{\text {in }}(T)=\operatorname{Tr}\left(\hat{\rho}_{\mathbf{k}}^{\text {in }} \hat{N}_{a_{\mathbf{k}}}^{\text {in }}\right)=\frac{1}{e^{\omega_{\mathbf{k}}^{\text {in }} / k T}-1} .
$$

Here, the Bogoliubov coefficients are

$$
\begin{aligned}
& \mu_{\mathbf{k}}(\infty)=i\left(\varphi_{\mathbf{k}}^{*}(\infty) \dot{\varphi}_{\mathbf{k}}^{\text {in }}-\dot{\varphi}_{\mathbf{k}}^{*}(\infty) \varphi_{\mathbf{k}}^{\text {in }}\right), \\
& \nu_{\mathbf{k}}(\infty)=i\left(\varphi_{\mathbf{k}}^{*}(\infty) \dot{\varphi}_{\mathbf{k}}^{\text {in } *}-\dot{\varphi}_{\mathbf{k}}^{*}(\infty) \varphi_{\mathbf{k}}^{\text {in } *}\right) .
\end{aligned}
$$

When there is a uniform magnetic field $B$ in addition to $E(t)$, each mode of the scalar field obeys the equation

$$
\ddot{\varphi}_{n k}(t)+\omega_{n k}^{2}(t) \varphi_{n k}(t)=0,
$$

where

$$
\omega_{n k}^{2}=\left(k_{z}+q E_{0} g(t)\right)^{2}+q B(2 n+1)+m^{2} .
$$

\section{TIME-DEPENDENT ELECTRIC FIELDS}

Pair production by localized electric fields in time or space significantly differs from that by the constant electric field due to a duration or a size effect $22,23,24,26,27]$. In this section we exploit an analytical method to calculate the Schwinger pair production rate by an electric field acting for a finite period of time or an oscillating electric field. This case is characterized by a homogeneous time-dependent electric field $E(t)$ with the maximum strength $E_{0}$ and the time scale $T$ defined as

$$
T=\frac{1}{2 E_{0}} \int_{-\infty}^{\infty} E(t) d t .
$$

The pair production rate is determined by two dimensionless parameters

$$
\epsilon=\frac{m}{q E_{0} T}, \quad \delta=\frac{q E_{0}}{\pi m^{2}} .
$$

Pair production is allowed for any $\epsilon$ but is strongly suppressed for $\epsilon \gg 1$.

The main result of Ref. 28] is that in the weak-field limit $\left(E<E_{c}\right)$ the mean number of boson pairs for each mode $\mathbf{k}$ per unit time and unit volume

$$
\mathcal{N}_{\mathbf{k}}=e^{-\mathcal{S}_{\mathbf{k}}}
$$

is determined by the WKB instanton action of Eq. (10)

$$
\mathcal{S}_{\mathbf{k}}=i \oint \sqrt{\left(k_{z}+q E_{0} g(t)\right)^{2}+m^{2}+\mathbf{k}_{\perp}^{2}} d t,
$$

where the integral is taken outside the contour in the complex plane of time. In Ref. [12] the production rate in scalar QED is defined as twice the imaginary part of the effective action

$$
w_{\mathbf{k}}=2 \ln \left(1+e^{-\mathcal{S}_{\mathbf{k}}}\right)=2 \sum_{n=1}^{\infty} \frac{(-1)^{n+1}}{n} e^{-n \mathcal{S}_{\mathbf{k}}} .
$$

The production rate (40) is even valid for very strong electric fields $\left(E \gg E_{c}\right)$ or $\left(\mathcal{S}_{\mathbf{k}} \ll 1\right)$. 
A few comments are in order. Taking into account spin statistics, the mean number of boson pairs with spin $s$ is given by 42 .

$$
\mathcal{N}_{\mathbf{k}}^{\text {boson }}=e^{w_{\mathbf{k}}^{\text {boson }}}-1=\left(1+e^{-\mathcal{S}_{\mathbf{k}}^{\text {boson }}}\right)^{2 s+1}-1,
$$

while the mean number of fermion pairs with spin $s$

$$
\mathcal{N}_{\mathbf{k}}^{\text {fermion }}=1-e^{-w_{\mathbf{k}}^{\text {fermion }}}=1-\left(1-e^{-\mathcal{S}_{\mathbf{k}}^{\text {fermion }}}\right)^{2 s+1},
$$

where $w_{\mathbf{k}}^{\text {boson/fermion }}$ is twice the imaginary part of the effective action for bosons and fermions, respectively, and $2 s+1$ is the spin multiplicity. The mean numbers without the spin multiplicity is also obtained in Ref. 43]. The mean numbers (41) and (42) hold even for very strong electric fields. The first term in Eq. (41) is the amplification factor for boson production. In the weak-field limit $\left(\mathcal{S}_{\mathbf{k}}>1\right)$, the mean number of boson or fermion pairs is approximately given by

$$
\mathcal{N}_{\mathbf{k}}^{\text {boson/fermion }} \approx(2 s+1) e^{-\mathcal{S}_{\mathbf{k}}^{\text {boson/fermion }}} .
$$

Another interesting point is the relation between the WKB instanton actions for bosons and fermions. For a Sauter electric field it is shown in Ref. [28] that the WKB instanton action for fermions together with the next-to-leading order is nothing but the WKB instanton action for bosons. Such a relation plausibly holds for any inhomogeneous electric field since the one-loop effective action for fermions

$$
S^{\text {fermion }}=-i \ln \operatorname{det}(i \mathbf{D}-m), \quad \mathbf{D}=\gamma_{\mu} \partial_{\mu}+i q A_{\mu},
$$

has the imaginary part, which is approximately for bosons

$$
\begin{aligned}
\operatorname{Im}\left(S^{\text {fermion }}\right) & =-\frac{i}{2}\left[\ln \operatorname{det}(i \mathbf{D}-m)+\ln \operatorname{det}(i \mathbf{D}-m)^{*}\right] \\
& \approx-\frac{i}{2} \ln \operatorname{det}\left(D^{2}+m^{2}\right) .
\end{aligned}
$$

Therefore, the WKB instanton action works for both scalar and spinor QED.

Now we develop an algorithm to compute the instanton action systematically. Introducing another variable

$$
\zeta=g(t)
$$

we rewrite Eq. (39) as

$$
\mathcal{S}_{\mathbf{k}}=i\left(q E_{0}\right) \oint \sqrt{\left(1+\frac{k_{z}}{q E_{0} \zeta}\right)^{2}+\frac{m^{2}+\mathbf{k}_{\perp}^{2}}{\left(q E_{0} \zeta\right)^{2}}} \frac{\zeta d \zeta}{g^{\prime}(\zeta)},
$$

and expand the square root in an inverse power series

$$
\sqrt{\left(1+\frac{k_{z}}{q E_{0} \zeta}\right)^{2}+\frac{m^{2}+\mathbf{k}_{\perp}^{2}}{\left(q E_{0} \zeta\right)^{2}}}=\sum_{n=0}^{\infty} \frac{\mathcal{C}_{n}}{\zeta^{n}},
$$

and the function $1 / g^{\prime}(\zeta)$ in a power series

$$
\frac{1}{g^{\prime}(\zeta)}=\sum_{n=0}^{\infty} \mathcal{D}_{n} \zeta^{n}
$$

Then the sum of negative residues of simple poles leads to the WKB instanton action

$$
\mathcal{S}_{\mathbf{k}}=2 \pi\left(q E_{0}\right) \sum_{n=0}^{\infty} \mathcal{C}_{n+2} \mathcal{D}_{n}
$$

The first few terms of $\mathcal{C}_{n}$ are

$$
\begin{aligned}
& \mathcal{C}_{0}=1, \quad \mathcal{C}_{1}=\alpha_{1}, \quad \mathcal{C}_{2}=\frac{\alpha_{2}}{2}, \\
& \mathcal{C}_{3}=-\frac{\alpha_{1} \alpha_{2}}{2}, \quad \mathcal{C}_{4}=\frac{\alpha_{1}^{2} \alpha_{2}}{2}-\frac{\alpha_{2}^{2}}{8},
\end{aligned}
$$

where

$$
\alpha_{1}=\frac{k_{z}}{q E_{0}}, \quad \alpha_{2}=\frac{m^{2}+\mathbf{k}_{\perp}^{2}}{\left(q E_{0}\right)^{2}} .
$$

The coefficients $\mathcal{D}_{n}$ are determined by the profile of $g(t)$. For specific models, we consider a Sauter-type electric field $E(t)=E_{0} \operatorname{sech}^{2}(t / T)$ and an oscillating electric field $E(t)=E_{0} \cos (t / T)$.

\section{A. Sauter-Type Electric Field}

The gauge potential for $E(t)=E_{0} \operatorname{sech}^{2}(t / T)$ in the $z$ direction is given by the Sauter potential

$$
A_{z}(t)=-E_{0} T \tanh \left(\frac{t}{T}\right)
$$

With the change of variable $\zeta=g(t)=T \tanh (t / T)$, we have the power series

$$
\frac{1}{g^{\prime}(t)}=\frac{1}{1-\frac{\zeta^{2}}{T^{2}}}=\sum_{n=0}^{\infty} \frac{\zeta^{2 n}}{T^{2 n}}
$$

and find $\mathcal{D}_{2 n}=1 / T^{2 n}$. This means that the WKB instanton action is

$$
\mathcal{S}_{\mathbf{k}}=2 \pi\left(q E_{0} T^{2}\right) \sum_{n=0}^{\infty} \frac{\mathcal{C}_{2 n+2}}{T^{2 n+2}} .
$$

In terms of the scaled variables and parameters

$$
\lambda \equiv \frac{k_{z}}{q E_{0} T}, \quad \kappa \equiv \frac{k_{\perp}}{m}, \quad Z=2 \pi q E_{0} T^{2}=\frac{2}{\delta \epsilon^{2}}
$$

the leading terms of the WKB instanton action are

$$
\mathcal{S}_{\mathbf{k}}=Z \epsilon^{2}\left(1+\kappa^{2}\right)\left[\frac{1}{2}+\frac{\lambda^{2}}{2}-\frac{\epsilon^{2}\left(1+\kappa^{2}\right)}{8}+\cdots\right] .
$$

In fact, the sum (55) can be done exactly as 28]

$$
\begin{aligned}
\mathcal{S}_{\mathbf{k}_{\perp}}= & \frac{Z}{2}\left[\sqrt{(1+\lambda)^{2}+\epsilon^{2}\left(1+\kappa^{2}\right)}\right. \\
& \left.+\sqrt{(1-\lambda)^{2}+\epsilon^{2}\left(1+\kappa^{2}\right)}-2\right] .
\end{aligned}
$$

The instanton method [28] gives much closer result to the exact one [21] than the worldline instantons [24]. 


\section{B. Oscillating Electric Field}

The oscillating electric field $E(t)=E_{0} \cos (t / T)$ has many physical applications such as laser fields [44, 45, 46]. The electric field from oscillating plasma due to pair production is approximately given by $E(t)=E \cos (t / T)$, where $E$ varies slowly during the oscillation period 47 , 48, 49, 50]. The gauge potential $\zeta=g(t)=T \sin (t / T)$ leads to the expansion

$$
\frac{1}{g^{\prime}(t)}=\frac{1}{\sqrt{1-\frac{\zeta^{2}}{T 2}}}=1+\frac{\zeta^{2}}{2 T^{2}}+\frac{3 \zeta^{4}}{8 T^{4}}+\frac{5 \zeta^{6}}{16 T^{6}}+\cdots
$$

Repeating the procedure in Sec. III A, we obtain the leading terms of the WKB instanton action

$$
\mathcal{S}_{\mathbf{k}}=Z \epsilon^{2}\left(1+\kappa^{2}\right)\left[\frac{1}{2}+\frac{\lambda^{2}}{4}-\frac{\epsilon^{2}\left(1+\kappa^{2}\right)}{16}+\cdots\right] .
$$

\section{INHOMOGENEOUS ELECTRIC FIELD}

For an inhomogeneous electric field localized in the $z$ direction, we may choose a Coulomb gauge $A_{0}(z)=$ $-E_{0} h(z)$, which leads to $E(z)=E_{0} h^{\prime}(z)$. Then the Klein-Gordon equation has the Fourier mode solution, $\Phi(\mathbf{x}, t)=e^{i \mathbf{k}_{\perp} \cdot \mathbf{x}_{\perp}-i \omega t} \phi_{\mathbf{k}_{\perp}}$, given by

$$
\left[-\frac{\partial^{2}}{\partial z^{2}}+m^{2}+\mathbf{k}_{\perp}^{2}-\left(\omega+q E_{0} g(z)\right)^{2}\right] \varphi_{\mathbf{k}_{\perp}}(z)=0 .
$$

The characteristic length scale $L$ is defined as

$$
L=\frac{1}{2 E_{0}} \int_{-\infty}^{\infty} E(z) d z
$$

As for time-dependent electric fields, two dimensionless parameters

$$
\bar{\epsilon}=\frac{m}{q E_{0} L}, \quad \delta=\frac{q E_{0}}{\pi m^{2}},
$$

determine the pair production rate. Remarkably the pair production rate is again given by the WKB instanton action 28]

$$
\mathcal{S}_{\mathbf{k}}=-i \oint \sqrt{\left(\omega+q E_{0} h(z)\right)^{2}-\left(m^{2}+\mathbf{k}_{\perp}^{2}\right)} d z,
$$

where the integral is taken outside the contour in the complex plane of space. We again introduce the variable

$$
\zeta=h(z),
$$

and rewrite Eq. (64) as

$$
\mathcal{S}_{\mathbf{k}}=-i\left(q E_{0}\right) \oint \sqrt{\left(1+\frac{\omega}{q E_{0} \zeta}\right)^{2}-\frac{m^{2}+\mathbf{k}_{\perp}^{2}}{\left(q E_{0} \zeta\right)^{2}}} \frac{\zeta d \zeta}{g^{\prime}(\zeta)} .
$$

The difference from the case of time-dependent electric fields is that the overall sign changes, and $\alpha_{1}$ and $\alpha_{2}$ are now replaced by $\bar{\alpha}_{1}=\omega /\left(q E_{0}\right)$ and $\bar{\alpha}_{2}=-\alpha_{2}$.

For specific models, we consider first a Sauter-type electric field $E(z)=E_{0} \operatorname{sech}^{2}(z / L)$ and then the electric field from strange stars in the next section. The Coulomb gauge is given by the Sauter potential

$$
A_{0}(z)=-E_{0} L \tanh \left(\frac{z}{L}\right) .
$$

With $\zeta=L \tanh (z / L)$ and the replacement of $\alpha_{1}$ by $\bar{\alpha}_{1}$ and $\alpha_{2}$ by $\bar{\alpha}_{2}=-\alpha_{2}$, the leading terms of the WKB instanton action are

$$
\mathcal{S}_{\mathbf{k}}=\bar{Z} \bar{\epsilon}^{2}\left(1+\kappa^{2}\right)\left[\frac{1}{2}+\frac{\bar{\lambda}^{2}}{2}+\frac{\bar{\epsilon}^{2}\left(1+\kappa^{2}\right)}{8}+\cdots\right] .
$$

where

$$
\bar{\lambda} \equiv \frac{\omega}{q E_{0} L}, \quad \kappa \equiv \frac{k_{\perp}}{m}, \quad \bar{Z}=2 \pi q E_{0} L^{2}=\frac{2}{\delta \bar{\epsilon}^{2}} .
$$

The exact sum of (55) is known 28]

$$
\begin{aligned}
\mathcal{S}_{\mathbf{k}_{\perp}=} & \frac{\bar{Z}}{2}\left[2-\sqrt{(1+\bar{\lambda})^{2}+\bar{\epsilon}^{2}\left(1+\kappa^{2}\right)}\right. \\
& \left.-\sqrt{(1-\bar{\lambda})^{2}+\bar{\epsilon}^{2}\left(1+\kappa^{2}\right)}\right] .
\end{aligned}
$$

\section{PAIR PRODUCTION FROM STRANGE QUARK STARS}

A source of the most strong electric fields beyond the critical strength is strange quark stars. A quark star, a hypothetical astrophysical object, could be formed from a hadron-quark phase transition at high densities and/or temperatures [51, 52, 53]. Chemical equilibrium of $u, d$ and $s$ quark and the charge neutrality of strange stars requires a net amount of electrons that are free to move the surface but bounded by the electric attraction from the positive core, thus forming an electrosphere of several hundred fermis. Then, the electrosphere of strange stars can generate an extremely strong electric field as strong as $5 \times 10^{17} \mathrm{~V} / \mathrm{cm}$, two order greater than the critical strength, and leads to efficient production of electronpositron pairs [13, 14, 15].

The static potential from the Thomas-Fermi model of the electron distribution at temperature $T$ [7]

$$
\begin{aligned}
& \frac{d^{2} V}{d z^{2}}=\frac{4 \alpha}{3 \pi}\left[\left(V^{3}-V_{q}^{3}\right)+\pi^{2} T^{2}\left(V-V_{q}\right)\right], \quad(z \leq 0) \\
& \frac{d^{2} V}{d z^{2}}=\frac{4 \alpha}{3 \pi}\left[V^{3}+\pi^{2} T^{2} V\right], \quad(z \geq 0),
\end{aligned}
$$

where $z$ is the coordinate normal to the quark surface, $\alpha$ is the fine structure constant, and $V_{q} / 3 \pi^{2}$ is the quark charge density inside the quark matter. The boundary 
condition is $V(-\infty)=V_{q}, V(\infty)=0$, and $V(0)=3 V_{q} / 4$. The Coulomb gauge potential is found [15]

$$
A_{0}(z)=\frac{\sqrt{2} \pi T}{\sinh \left[2 \sqrt{\frac{\alpha \pi}{3}} T\left(z+z_{0}\right)\right]},
$$

and the electric field is

$$
E(z, T)=\sqrt{\frac{8 \pi^{3}}{3}} T^{2} \frac{\cosh \left[2 \sqrt{\frac{\alpha \pi}{3}} T\left(z+z_{0}\right)\right]}{\sinh ^{2}\left[2 \sqrt{\frac{\alpha \pi}{3}} T\left(z+z_{0}\right)\right]},
$$

whose characteristic scales are

$$
E_{0}=\sqrt{\frac{8 \pi^{3} \alpha}{3}} T^{2}, \quad L=\sqrt{\frac{3}{\alpha \pi}} \frac{1}{2 T} .
$$

The higher (lower) the temperature is, the greater (smaller) is the maximum strength and the narrower (wider) is the width of the electric field.

With

$$
\zeta=-\frac{L}{\sinh \left[\frac{z+z_{0}}{L}\right]},
$$

the WKB instanton action becomes

$$
\begin{aligned}
\mathcal{S}_{\mathbf{k}}= & -i\left(q E_{0} L\right) \oint \sqrt{\left(1+\frac{\omega}{q E_{0} \zeta}\right)^{2}-\frac{m^{2}+\mathbf{k}_{\perp}^{2}}{\left(q E_{0} \zeta\right)^{2}}} \\
& \times \frac{d \zeta}{\sqrt{1+\frac{\zeta^{2}}{L^{2}}}} .
\end{aligned}
$$

Thus, in terms of the scaled variables and parameters in Sec. IV, the leading terms of the WKB instanton action are

$$
\begin{aligned}
\mathcal{S}_{\mathbf{k}} & =2 \pi\left(q E_{0} L^{2}\right)\left[\frac{\omega}{q E_{0} L}-\frac{1}{4} \frac{\omega}{q E_{0} L} \frac{m^{2}+\mathbf{k}_{\perp}^{2}}{\left(q E_{0} L\right)^{2}}+\cdots\right] \\
& =\bar{Z} \bar{\lambda}\left[1-\frac{\bar{\epsilon}^{2}\left(1+\kappa^{2}\right)}{4}+\cdots\right] .
\end{aligned}
$$

Then, the mean number (42) of electron-positron pairs per unit time and volume

$$
\mathcal{N}_{\mathbf{k}}^{\text {fermion }}=2 e^{-\mathcal{S}_{\mathbf{k}}}-e^{-2 \mathcal{S}_{\mathbf{k}}}
$$

is the spectrum of emitted pairs. As $\bar{Z} \bar{\lambda}=\sqrt{3 \pi / \alpha} \times$ $(\omega / T)$, hot strange stars produce more pairs of electrons and positrons than cold ones, confirming the numerical result of Ref. [15].

\section{CONCLUSION}

In this talk, we critically reviewed the Schwinger mechanism at zero or finite temperature in inhomogeneous electric fields motivated by astrophysics or terrestrial experiments. As exact solutions of the Klein-Gordon or Dirac equation minimally coupled to inhomogeneous electromagnetic fields are known only for a few cases, for general electromagnetic fields, however, one has to rely on some approximation schemes. Inhomogeneous electric fields result in a finite size or duration effect and differs from that by a constant field 22, 23, 24]. We applied the phase-integral method to find the WKB instanton action for the mode equations in inhomogeneous electromagnetic fields and then calculated the pair production rate by a Sauter-type electric field either in space or time 26, 27, 28] and an oscillating electric field. We also studied the thermal effect on pair production by an electric field that acts for a finite period of time [29]. Finally, we applied the WKB instanton action method to strange stars to calculate the electron-positron pair production rate.

The issues not treated in this talk are the effective action and the back reaction of QED at zero or finite temperature. It is a complicated task to obtain the effective action in inhomogeneous electromagnetic fields. In canonical quantum field theory, we may follow Ref. [54], according to which the effective action is related with the scattering amplitude as

$$
\left.e^{i S_{\text {eff }}}=e^{i \int d t d^{3} \mathbf{x} \mathcal{L}_{\text {eff }}}=\langle 0, \text { out }| 0, \text { in }\right\rangle .
$$

Thus the effective action requires a complete knowledge of evolution of the ingoing vacuum to the outgoing vacuum, which may follow from the vacuum wave functional from Eqs. (25) and (26) for each mode. Another important issue is the QED back reaction problem, which is described by, for instance, the scalar QED action of the form

$$
\mathcal{L}=\phi^{*}\left(\partial_{\mu}+i q A_{\mu}\right)^{2} \phi-\frac{1}{4} F_{\mu \nu} F^{\mu \nu} .
$$

The back reaction cannot be neglected for strong electric fields because the additional electric field produced by pairs is comparable to the applied field. In fact, positive (negative) charges of produced pairs move in the same (opposite) direction of the applied electric field, so the current due to pairs induces an electric field in the opposite direction of the applied field and overshoots it until the process is reversed, which leads to the famous plasma oscillation [47, 48, 49, 50]. These issues will addressed in a future publication [55].

\section{Acknowledgments}

The author would like to thank Hyun Kyu Lee and Don N. Page for collaborations and useful discussions and Professor S. P. Gavrilov and Professor Tiberiu Harko for comments on the WKB approximation and Holger Gies and Professor Naoki Itoh for useful information. And he also would like to thank the warm hospitality of ICRANet during the 10th Italian-Korean Symposium on Relativistic Astrophysics, Pescara, June 25-30, 2007. 
This work was supported by the Korea Science and Engineering Foundation (KOSEF) grant funded by the Korea government (MOST) (No. R01-2005-10404-0).
[1] G. V. Dunne, "Heisenberg-Euler Effective Lagrangians: Basics and Extensions," From Fields to Strings: Circumnavigating Theoretical Physics, edited by M. Shifman, A. Vainshtein, and J. Wheater, (World Scientific, Singapore, 2005), Vol. I, pp. 445-522, hep-th/0406216

[2] E. S. Fradkin, D. M. Gitman, and Sh. M. Shvartsman, QED with Unstable Vacuum (Springer-Verlag, Berlin, 1991).

[3] SLAC Linac Coherent Light Source, 2007, http://www-ssrl.slac.stanford.edu/lcls.

[4] DESY X-Ray Free Electron Laser, 2007, http://xfel.desy de.

[5] A. Ringwald, Phys. Lett. B 510, 107 (2001); "Fundamental Physics at an X-Ray Free Electron Laser," Electromagnetic Probes of Fundamental Physics, edited by W. Marciano and S. White (World Scientific, Singapore, 2003), pp. 63-74, hep-ph/0112254 "Boiling the Vacuum with an X-Ray Free Electron Laser," Quantum Aspects of Beam Physics 2003, edited by P. Chen and K. Reil (World Scientific, Singapore, 2004), pp. 149-163, hep-ph/0304139

[6] P. M. Wood and C. Thompson, "Soft Gamma Repeaters and Anomalous X-ray Pulsars: Magnetar Candiates," in Compact Stellar X-ray Sources, edited by W. H. G. Lewin and M. van der Klis (Cambridge Univ., Cambridge, 2006), pp 547-586, astrop-ph/0406133

[7] C. Alcock, E. Farhi, and A. V. Olinto, Astrophys. J. 310, 261 (1986).

[8] P. Haensel, J. L. Zdunik, and R. Schaeffer, Astron. Astrophys. 160, 121 (1986).

[9] F. Weber, Prog. Part. Nucl. Phys. 54, 193 (2005).

[10] A. K. Harding and D. Lai, Rep. Prog. Phys. 69, 2631 (2006).

[11] M. Marklund and P. K. Shukla, Rev. Mod. Phys. 78, 591 (2006).

[12] J. Schwinger, Phys. Rev. 82, 664 (1951).

[13] V. V. Usov, Phys. Rev. Lett. 80, 230 (1998).

[14] V. V. Usov, T. Harko, and K. S. Cheng, Astrophys. J. 620, 915 (2005).

[15] T. Harko and K. S. Cheng, Astrophys. J. 643, 318 (2006).

[16] R. C. Duncan, "Physics in Ultra-strong Magnetic Fields," astro-ph/0002442 (2000).

[17] H. K. Lee and Y. Yoon, JHEP 0603, 078 (2006); JHEP 0703, 086 (2007).

[18] W. Heisenberg and H. Euler, Z. Physik 98, 714 (1936).

[19] V. Weisskopf, K. Dan. Vidensk. Selsk. Mat. Fys. Medd. XIV, 6 (1936).

[20] R. Ruffini and S.-S. Xue, J. Korean Phys. Soc. 49, 715 (2006).

[21] A. I. Nikishov, Sov. Phys. JETP 30, 660 (1970); Nucl. Phys. B 21, 346 (1970).

[22] R.-C. Wang and C.-Y. Wong, Phys. Rev. D 38, 348 (1988).

[23] G. V. Dunne and C. Schubert, Phys. Rev. D 72, 105004 (2005).

[24] G. V. Dunne, Q.-H. Wang, H. Gies, and C. Schubert, Phys. Rev. D 73, 065028 (2006).

[25] N. Fröman and P. O. Fröman, Nucl. Phys. A147, 606
(1970); Phase-Integral Method (Springer-Verlag, New York, 1996).

[26] S. P. Kim and D. N. Page, Phys. Rev. D 65, 105002 (2002).

[27] S. P. Kim and D. N. Page, Phys. Rev. D 73, 065020 (2006).

[28] S. P. Kim and D. N. Page, Phys. Rev. D 75, 045013 (2007).

[29] S. P. Kim and H. K. Lee, Phys. Rev. D 76, 125002 (2007).

[30] L. Parker, Phys. Rev. Lett. 21, 562 (1968); Phys. Rev. 183, 1057 (1969).

[31] B. S. DeWitt, Phys. Rep. 19, 295 (1975).

[32] H. R. Lewis, Jr. and W. B. Riesenfeld, J. Math. Phys. 10, 1458 (1969).

[33] S. P. Kim and C. H. Lee, Phys. Rev. D 62, 125020 (2000); S. Sengupta, F. C. Khanna, and S. P. Kim, Phys. Rev. D 68, 105014 (2003).

[34] W. Dittrich, Phys. Rev. D 19, 2385 (1979).

[35] S. P. Gavrilov, D. M. Gitman, and E. S. Fradkin, Yad. Fiz. 46, 172 (1987) [Sov. J. Nucl. Phys. 46, 107 (1987)].

[36] P. Elmfors, D. Persson, and B.-S. Skagerstam, Phys. Rev. Lett. 71, 480 (1993); P. Elmfors, P. Liljenberg, D. Persson, and B.-S. Skagerstam, Phys. Rev. D 51, 5885 (1995).

[37] M. Loewe and J. C. Rojas, Phys. Rev. D 46, 2689 (1992).

[38] A. K. Ganguly, P. K. Kaw, and J. C. Parikh, Phys. Rev. C 51, 2091 (1995).

[39] J. Hallin and P. Liljenberg, Phys. Rev. D 52, 1150 (1995).

[40] P. Elmfors and B.-S. Skagerstam, Phys. Lett. B 348, 141 (1995).

[41] H. Gies, Phys. Rev. D 60, 105002 (1999); Phys. Rev. D 61, 085021 (2000).

[42] S. P. Kim and D. N. Page, Nuovo Cimento 120 B, 1193 (2005).

[43] S. P. Gavrilov, D. M. Gitman, and J. L. Tomazelli, Nucl. Phys. B, (2008) in press arXiv:hep-th/0612064.

[44] E. Brezin and C. Itzykson, Phys. Rev. D 2, 1191 (1970).

[45] V. S. Popov, Sov. Phys. JETP 34, 709 (1972); Sov. Phys. JETP 35, 659 (1972); V. S. Popov and M. S. Marinov, Yad. Fiz. 16, 809 (1972) [Sov. J. Nucl. Phys. 16, 449 (1973)].

[46] V. S. Popov, Phys. Lett. A 298, 83 (2002).

[47] Y. Kluger, J. M. Eisenberg, B. Svetitsky, F. Cooper, and E. Mottola, Phys. Rev. Lett. 67, 2427 (1991); Phys. Rev. D 45, 4659 (1992).

[48] F. Cooper and E. Mottola, Phys. Rev. D 40, 456 (1989).

[49] R. Ruffini, L. Vitagliano, and S.-S. Xue, Phys. Lett. B 559, 12 (2003).

[50] R. Ruffini, G. V. Vereshchagin, and S.-S. Xue, Phys. Lett. A 371, 399 (2007).

[51] N. Itoh, Prog. Theor. Phys. 44, 291 (1970).

[52] A. R. Bodmer, Phys. Rev. D 4, 1601 (1971).

[53] E. Witten, Phys. Rev. D 30, 272 (1984).

[54] J. Ambjorn, R. J. Hughes, and N. K. Nielsen, Ann. Phys. 150, 92 (1983).

[55] S. P. Kim and H. K. Lee, "Effective Action and Back Reaction of QED at Finite temperature," in prepartion. 